\title{
GALACTIC DUST DISTRIBUTION IN \\ THE SOLAR NEIGHBORHOOD
}

\author{
J. H. CAHN and R.D. NOSEK \\ University of Illinois Observatory, Urbana, IIl. 6180I, U.S.A.
}

\begin{abstract}
Using the Shklovsky-Minkowski-Aller constant mass method of distances to planetary nebulae, a model of the Galactic dust distribution in the vicinity of the Sun has been determined. Distances are determined in part from extinctions derived from radio continuum to $H \beta$ flux ratios for an assumed electron temperature of $7000 \mathrm{~K}$. The distance scale is based on the brightness of planetary nebulae in the Magellanic Clouds (Seaton, 1968).
\end{abstract}

In an extension of the work of Cahn and Kaler (1971) an empirically determined dust distribution in the vicinity of the Sun has been developed. The overall features of the dust distribution are based upon optical color excess measurements and $21 \mathrm{~cm}$ hydrogen position determinations. A recently revised set of $\mathrm{H} \beta$ extinctions was then used to refine the positions of the dust.

In the Cahn and Kaler paper, hereinafter referred to as CK, a Mills (1959) spiral was picked whose parameters gave the best agreement with the then known $\mathrm{H} \beta$ extinctions. It was realized at that time that such an idealization of the local spiral arms was too inflexible to permit accurate predictions of extinctions over the accessible regions of the Galaxy. It was then decided to try to develop an empirical dust map, which would provide the desired flexibility. The first step in constructing the map was to initialize the model with existing measurements. We used the color excess map developed by Fitzegerald (1968) as shown in Figure 1 and the $21 \mathrm{~cm}$ maps of Winnberg (1968) shown in Figure 2 and that of Kerr and Weaver as given by Simonson (1970) in Figure 3. In order to refine these positions we then extended and refined the extinctions in CK as given in Table I. These values are based upon an electron temperature of $7000 \mathrm{~K}$ rather than $5000 \mathrm{~K}$ as suggested both by Kaler and by Peimbert (1971). The radio fluxes include unpublished measurements by Cahn, Rubin and Hermann, and Aller and Milne in addition to those already reported in CK.

The resulting adjusted map is shown in Figure 4. In order to store such a large fraction of the Galaxy on the computer, only two levels of extinction, $0.407 \mathrm{kpc}^{-1}$ and $2.033 \mathrm{kpc}^{-1}$ were used, the latter being the shaded area in Figure 4. To account for the finite thickness of the dust distribution, the values of extinction in the plane were reduced by the Gaussian factor $\exp \left[-(z / 150 \mathrm{pc})^{2}\right]$ where $z$ is height above or below the plane. In homogeneous media of either the low or high specific extinction, the integrals to infinity along the line of sight give extinctions of $0.061 \mathrm{cscb}_{\mathrm{II}}$ and $0.305 \mathrm{cscb}_{\mathrm{II}}$ respectively, where $b_{\mathrm{II}}$ is the galactic latitude. Figure 4 represents a fit to the measured extinctions resulting in a correlation coefficient of 0.99 .

It is to be understood that with such a limited list of calibrators, each of whose distance is only statistically accurate, that the map is still very speculative. In Figure 4, 
TABLE I

Galactic coordinates

\begin{tabular}{|c|c|c|c|c|c|c|}
\hline \multicolumn{2}{|c|}{ Nebula } & \multirow{2}{*}{$\frac{l_{\mathrm{II}}}{0.38}$} & \multirow{2}{*}{$\frac{b_{11}}{12.22}$} & \multirow{2}{*}{$\begin{array}{l}\text { Distance }(\mathrm{pc}) \\
3680\end{array}$} & \multirow{2}{*}{$\begin{array}{l}\text { Radius }(\mathrm{pc}) \\
0.075\end{array}$} & \multirow{2}{*}{$\begin{array}{l}\text { Extinction } \\
0.61\end{array}$} \\
\hline IC & 4634 & & & & & \\
\hline NGC & 6369 & 2.44 & 5.85 & 1040 & 0.070 & 2.21 \\
\hline NGC & 6620 & 5.88 & -6.15 & 6360 & 0.077 & 1.49 \\
\hline NGC & 6445 & 8.07 & 3.90 & 1360 & 0.109 & 1.27 \\
\hline NGC & 6309 & 9.66 & 14.81 & 2800 & 0.094 & 0.93 \\
\hline IC & 4593 & 25.41 & 40.73 & 2990 & 0.093 & 0.14 \\
\hline NGC & 6818 & 25.86 & -17.90 & 1970 & 0.087 & 0.20 \\
\hline NGC & 6751 & 29.23 & -5.93 & 2570 & 0.131 & 0.52 \\
\hline NGC & 6778 & 34.61 & -6.71 & 2850 & 0.109 & 0.68 \\
\hline NGC & 6572 & 34.62 & 11.84 & 1670 & 0.058 & 0.44 \\
\hline NGC & 7293 & 36.24 & -57.10 & 150 & 0.293 & 0.05 \\
\hline NGC & 7009 & 37.76 & -34.58 & 1270 & 0.087 & 0.20 \\
\hline CN3-1 & & 38.26 & 12.09 & 4910 & 0.064 & 0.59 \\
\hline NGC & 6781 & 41.84 & -2.98 & 670 & 0.173 & 1.27 \\
\hline NGC & 6210 & 43.12 & 37.76 & 2060 & 0.081 & 0.15 \\
\hline NGC & 6804 & 45.75 & -4.59 & 1640 & 0.095 & 1.00 \\
\hline NGC & 6879 & 57.23 & -8.93 & 6750 & 0.082 & 0.65 \\
\hline NGC & 6886 & 60.14 & -7.74 & 3980 & 0.073 & 1.15 \\
\hline NGC & 6853 & 60.83 & -3.69 & 260 & 0.211 & 0.10 \\
\hline NGC & 6720 & 63.15 & 13.98 & 840 & 0.141 & 0.21 \\
\hline NGC & 6842 & 65.91 & 0.60 & 1560 & 0.180 & 1.01 \\
\hline NGC & 6894 & 69.48 & -2.62 & 1510 & 0.162 & 0.92 \\
\hline NGC & 7027 & 84.92 & -3.49 & 1210 & 0.041 & 1.54 \\
\hline HU1-2 & & 86.54 & -8.83 & 5350 & 0.065 & 0.75 \\
\hline NGC & 7354 & 107.84 & 2.31 & 1640 & 0.080 & 1.86 \\
\hline NGC & 40 & 120.02 & 9.87 & 1180 & 0.104 & 0.90 \\
\hline IC & 3568 & 123.66 & 34.55 & 2520 & 0.110 & 0.34 \\
\hline IC & 289 & 138.82 & 2.81 & 1430 & 0.128 & 1.49 \\
\hline NGC & 1501 & 144.56 & 6.55 & 1150 & 0.144 & 1.11 \\
\hline NGC & 1514 & 165.53 & -15.29 & 720 & 0.120 & 0.63 \\
\hline NGC 2 & 23712 & 189.16 & 19.83 & 1670 & 0.176 & 0.22 \\
\hline $\mathbf{J}$ & 320 & 190.39 & -17.77 & 5050 & 0.079 & 0.72 \\
\hline NGC & 2022 & 196.68 & -10.93 & 2640 & 0.124 & 0.47 \\
\hline NGC & 2392 & 197.88 & 17.40 & 1190 & 0.129 & 0.35 \\
\hline NGC & 1535 & 206.48 & -40.57 & 2230 & 0.099 & 0.12 \\
\hline IC & 418 & 215.22 & -24.27 & 1740 & 0.053 & 0.33 \\
\hline IC & 2165 & 221.33 & -12.40 & 3520 & 0.068 & 0.85 \\
\hline NGC & 2440 & 234.84 & 2.43 & 1360 & 0.109 & 0.63 \\
\hline NGC & 2610 & 239.64 & 13.95 & 1810 & 0.151 & 0.77 \\
\hline NGC & 3242 & 261.06 & 32.06 & 1040 & 0.094 & 0.30 \\
\hline NGC & 2818 & 261.98 & 8.60 & 1750 & 0.170 & 0.67 \\
\hline NGC & 3132 & 272.11 & 12.39 & 1220 & 0.133 & 0.28 \\
\hline NGC & 4361 & 294.11 & 43.62 & 940 & 0.186 & 0.17 \\
\hline NGC & 5307 & 312.38 & 10.56 & 2980 & 0.091 & 0.76 \\
\hline IC & 4406 & 319.69 & 15.74 & 2140 & 0.104 & 0.47 \\
\hline NGC & 5882 & 327.84 & 10.09 & 2230 & 0.076 & 0.44 \\
\hline NGC & 6326 & 338.20 & -8.38 & 2930 & 0.086 & 0.81 \\
\hline NGC & 6153 & 341.84 & 5.46 & 1390 & 0.083 & 1.26 \\
\hline NGC & 6072 & 342.16 & 10.83 & 990 & 0.168 & 1.15 \\
\hline NGC & 6563 & 358.50 & -7.33 & 1400 & 0.146 & 0.62 \\
\hline
\end{tabular}




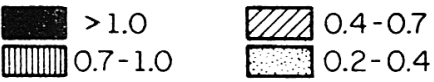

$\square 0.0-0.2$

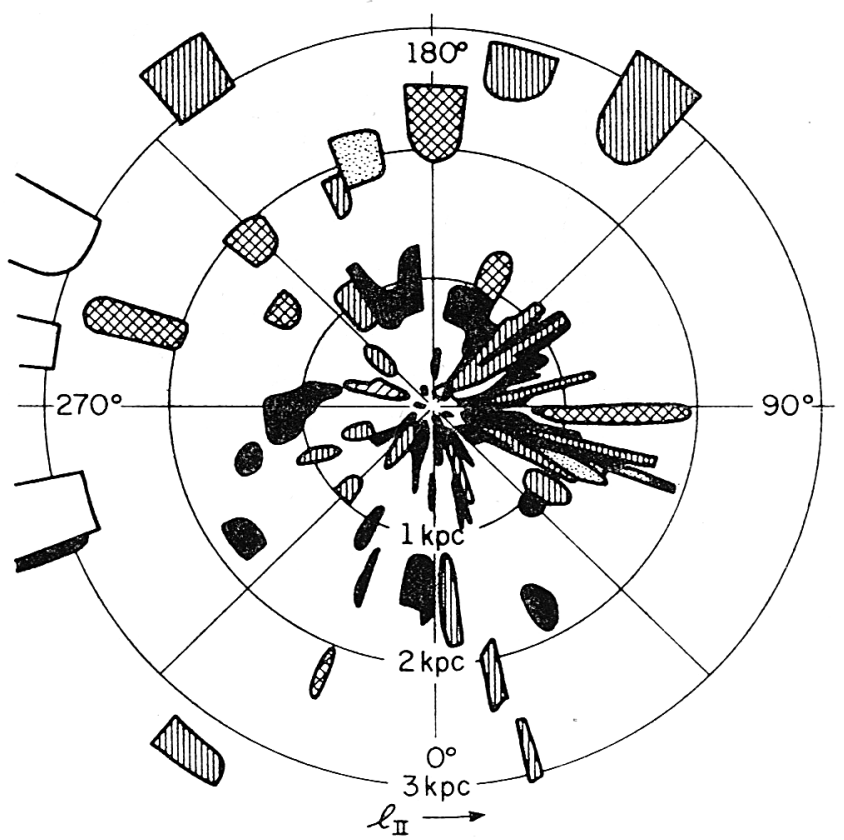

Fig. 1. Color excesses of Fitzgerald (1968). Darkened areas indicate excesses greater than 1 mag. $\mathrm{kpc}^{-1}$.

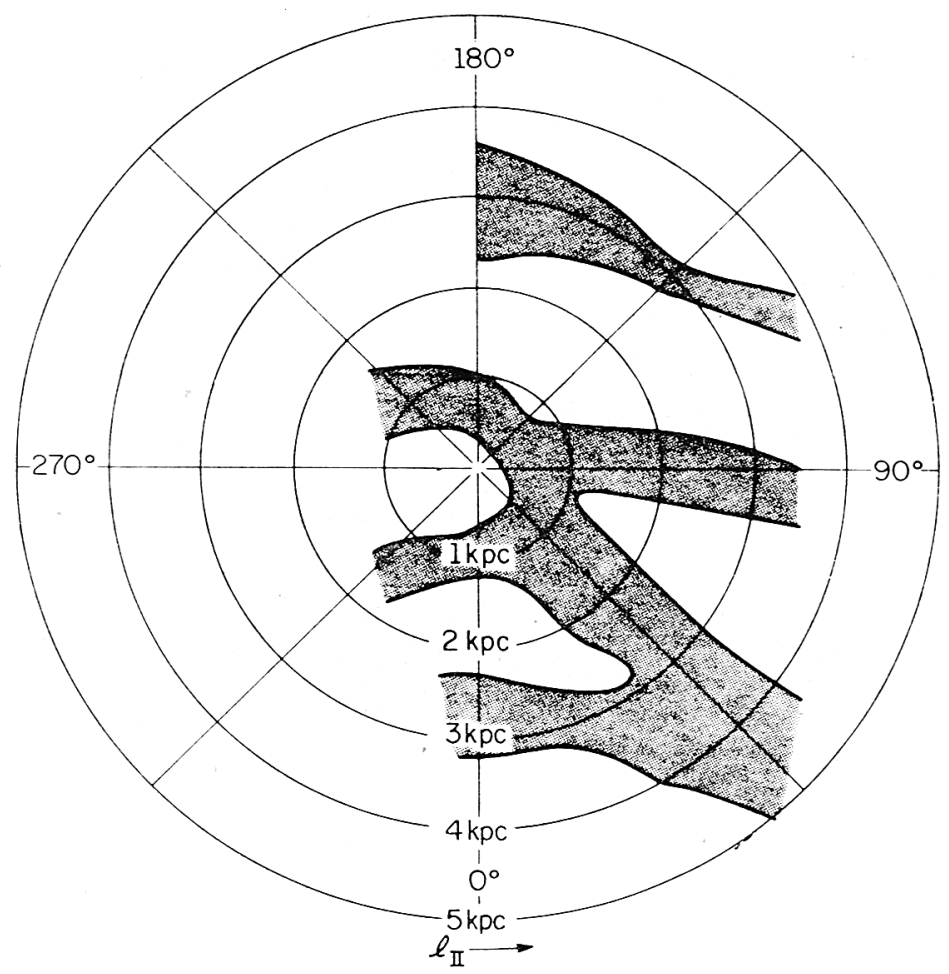

Fig. 2. Part of $21 \mathrm{~cm}$ map of Winnberg (1968) 


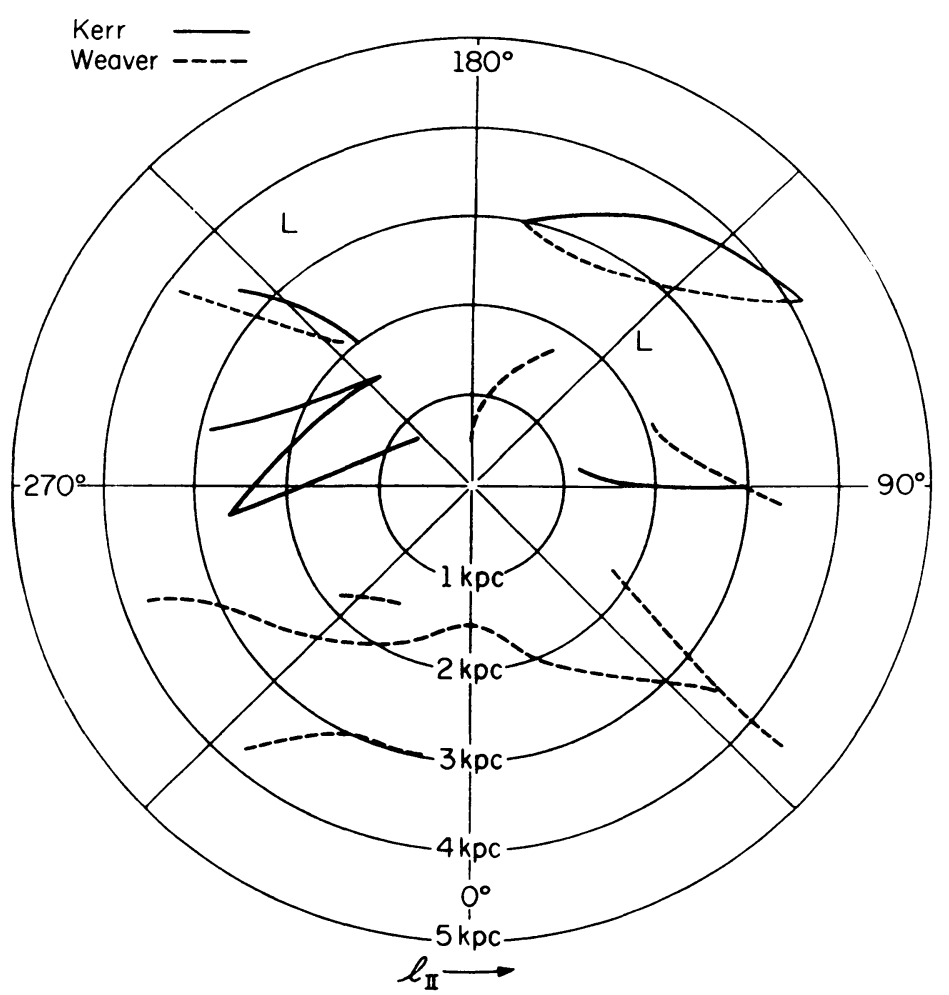

Fig. 3. Part of $21 \mathrm{~cm}$ map of Kerr and Weaver data as given in Simonson (1970).

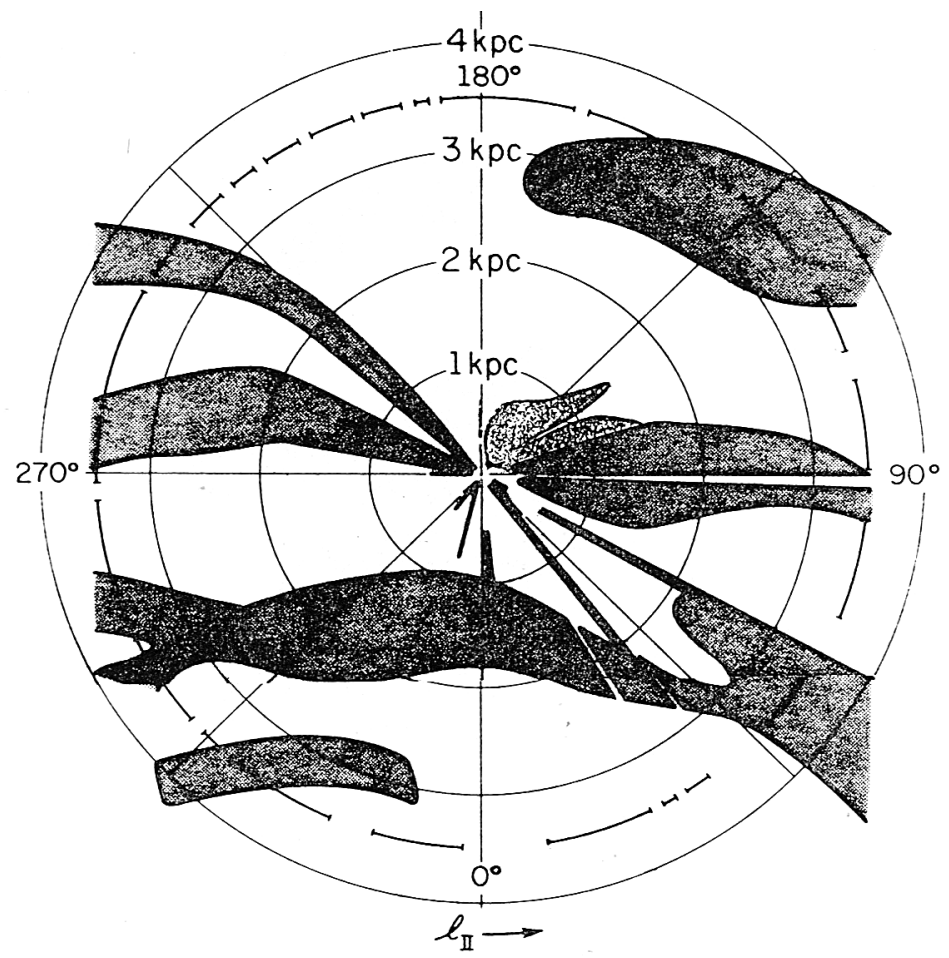

Fig. 4. Adjusted map of dust distribution in accord with planetary nebular extinctions and distances. Broken line indicates coverage (c.f. text).
d online by Cambridge University Press 
the intermittent line at $3.5 \mathrm{kpc}$ represents the incompleteness of the data. The solid part of the line represents uncalibrated regions of the map. In addition, regions closest to the Sun are most reliable, since the average value of $b_{\mathrm{II}}$ is $15^{\circ}$.

Finally, it is to be hoped that the present work can be coordinated with determinations of color excess to help determine $R$, the ratio of total to selective absorption.

\section{References}

Cahn, J. H. and Kaler, J. B.: 1971, Astrophys. J. Suppl. 22, 319.

Fitzgerald, M. Pim: 1968, Astron. J. 73, 983.

Mills, B.: 1959, in R. N. Bracewell (ed.), 'Paris Symposium on Radio Astronomy', IAU Symp. 9, 431.

Peimbert, M.: 1971, Bol. Obs. Tonantzintla Tacubaya 6, 29.

Seaton, M. J.: 1968, Astrophys. Letters 2, 55.

Simonson, S. C.: 1970, Astron. Astrophys. 9, 163. 